\title{
Algorithmic Approach to Non-symmetric 3-class Association Schemes
}

\author{
Leif K. Jørgensen \\ Dept. of Mathematical Sciences, Aalborg University \\ Fr. Bajers Vej 7, 9220 Aalborg, Denmark. \\ leif@math.aau.dk
}

Summary. There are 24 feasible parameter sets for a primitive non-symmetric association schemes with 3 classes and at most 100 vertices. Using computer search, we prove non-existence for three feasible parameter sets. Eleven cases are still open.

In the imprimitive case, we survey the known results including some constructions of infinite families of schemes. In the smallest case that has been open up to now, we use computer search to find new schemes. These schemes are equivalent to "skew" Bush-type Hadamard matrices of order 36. We also consider directed graphs that satisfy only some of the conditions required for a non-symmetric association scheme with 3 classes.

\section{Introduction}

The theory of association schemes was for a long time concentrated on the investigation of the symmetric association schemes generated by distance regular graphs. In this context the symmetric association schemes with two classes are exactly the schemes generated by strongly regular graphs.

More opportunities appear as soon as we are dealing with at least three classes. A good survey of symmetric association schemes with three classes was provided by van Dam [6].

In this paper we consider non-symmetric association schemes with three classes. From each such association scheme, a symmetric association scheme with two classes can be obtained by merging the non-symmetric relations. Feasibility conditions for the existence of these association schemes have previously been considered by Bannai and Song [2], Song [35] and by Goldbach and Claasen [13].

In this paper we make an attempt of a more systematic investigation of non-symmetric 3-class association schemes with a relatively small number of vertices. In the primitive case we generate all feasible parameter sets with at most 100 vertices. There are 24 such parameter sets. We review known results and prove non-existence results for three parameter sets, while 11 cases still remain open. 
We also briefly consider normally regular digraphs (in the sense of [24]) as a generalization of non-symmetric 3-class association schemes.

For the imprimitive case we start from a consideration of doubly regular $(m, r)$-team tournament in the sense of [26]. In [26] we distinguish three possible types of such directed graphs. A graph of type 3 can not be a relation of an association scheme, however we do not know if any graph of this type exists. Types 1 and 2 indeed correspond to imprimitive non-symmetric 3 -class association schemes. Graphs of type 1 are easily reduced to doubly regular tournaments in the sense of [33]. Thus we concentrate on graphs of type 2 and the corresponding association schemes. In particular we consider a subtype of type 2 which has links to Bush-type Hadamard matrices.

Here we investigate the smallest open case of order 36 . We find four such association schemes by computer search, but we leave open the problem of complete enumeration of all association schemes with this set of parameters. We expect that there may be a large number of such schemes - probably all with small automorphism groups. N. Ito [19] has proved that they can not have an automorphism group of rank 4.

It should be stressed that our approach is strictly algorithmic, essentially depending on the use of computers. The computer is used already on the initial stage of the generation of all feasible sets of parameters of primitive schemes.

For computer-aided constructive enumeration of all association schemes with a given set of parameters we use two different approaches. The first approach makes use of a complete catalogue of strongly regular graphs with the parameters that would be obtained by merging the non-symmetric relations. Such complete catalogues have been constructed by Coolsaet, Degraer and Spence [5] and by Hoffman and Singleton [16]. The second approach is an orderly generation algorithm in the spirit of Faradžev [8] and Read [32].

These techniques are used to exclude existence of three feasible parameter sets for primitive association schemes. The second technique was also used to find the above mentioned imprimitive association schemes of order 36 . In that case the search space is huge and it was not possible to complete the full search. But we successfully used some ad hoc tricks in order to catch in the whole search space a few lucky directions leading to a construction of the desired combinatorial objects.

We hope that the results presented in this paper may help to promote further approaches towards constructive enumeration of association schemes.

\section{Preliminaries}

Let $X$ be a finite set $(|X|=v)$ and let $\left\{R_{0}, R_{1}, \ldots, R_{d}\right\}$ be a partition of $X \times X$. Then we say that $\mathcal{X}=\left(X,\left\{R_{0}, R_{1}, \ldots, R_{d}\right\}\right)$ is an association scheme with $d$ classes if the following conditions are satisfied

- $R_{0}=\{(x, x) \mid x \in X\}$, 
- $\quad$ for each $i, R_{i}^{\mathrm{t}}:=\left\{(x, y) \mid(y, x) \in R_{i}\right\}=R_{i^{\prime}}$, for some $i^{\prime}$,

- and for each triple $(i, j, h), i, j, h \in\{0, \ldots, d\}$ there exists a so-called intersection number $p_{i j}^{h}$ such that for all $x, y \in X$ with $(x, y) \in R_{h}$ there are exactly $p_{i j}^{h}$ elements $z \in X$ so that $(x, z) \in R_{i}$ and $(z, y) \in R_{j}$.

For $i>0$ the relation $R_{i}$ can be viewed as the edge set of the (undirected or directed) graph $\left(X, R_{i}\right)$. We will frequently identify this graph with the relation $R_{i}$.

If $i=i^{\prime}$ for all $i$ then $\mathcal{X}$ is said to be symmetric, otherwise it is nonsymmetric. If the graphs $R_{1}, \ldots, R_{d}$ all are connected then we say that $\mathcal{X}$ is primitive, otherwise it is imprimitive.

In this paper we consider non-symmetric association schemes with $d=3$ classes. We will assume that the relations are enumerated so that $R_{1}$ and $R_{2}$ are non-symmetric, $R_{2}=R_{1}^{\mathrm{t}}$, and $R_{3}$ is a symmetric relation. In this case the association scheme is determined uniquely by relation $R_{1}$.

If $A$ denotes the adjacency matrix of the relation $R_{1}$ then the adjacency matrices of $R_{0}, R_{2}$ and $R_{3}$ are $I, A^{\mathrm{t}}$ and $J-I-A-A^{\mathrm{t}}$, respectively. The BoseMesner algebra of $\mathcal{X}$ is the matrix algebra $\mathcal{A}$ spanned by these four matrices, see Bannai and Ito [1].

Higman [15] proved that an association scheme with $d \leq 4$ has a commutative Bose-Mesner algebra, which means that $p_{i j}^{h}=p_{j i}^{h}$, for all $i, j, h$.

Thus multiplication in the Bose-Mesner algebra is determined by the following equations.

$$
\begin{array}{r}
A J=J A=\kappa J \\
A A^{\mathrm{t}}=\kappa I+\lambda\left(A+A^{\mathrm{t}}\right)+\mu\left(J-I-A-A^{\mathrm{t}}\right) \\
A^{\mathrm{t} A}=\kappa I+\lambda\left(A+A^{\mathrm{t}}\right)+\mu\left(J-I-A-A^{\mathrm{t}}\right) \\
A^{2}=\alpha A+\beta A^{\mathrm{t}}+\gamma\left(J-I-A-A^{\mathrm{t}}\right),
\end{array}
$$

where $\kappa=p_{12}^{0}, \lambda=p_{12}^{1}=p_{21}^{1}=p_{12}^{2}=p_{21}^{2}, \mu=p_{12}^{3}=p_{21}^{3}, \alpha=p_{11}^{1}, \beta=p_{11}^{2}$ and $\gamma=p_{11}^{3}$.

We note that $\alpha=\lambda$. This is seen by counting in two ways the pairs $(y, z)$ so that $(x, y),(x, z),(y, z) \in R_{1}$, for a fixed vertex $x$.

Since $\mathcal{A}$ is commutative and consists of normal matrices, the matrices of $\mathcal{A}$ have a common diagonalization, i.e., $\mathcal{A}$ has a basis $\left\{E_{0}, E_{1}, E_{2}, E_{3}\right\}$ of orthogonal projections.

A relation (say $R_{1}$ ) of a symmetric association scheme with two classes is a strongly regular graph with parameters $(v, k, a, c)$, where $v=|X|, k=$ $p_{11}^{0}, a=p_{11}^{1}, c=p_{11}^{2}$. And conversely, if $R_{1}$ is a strongly regular graph and $R_{2}$ is the complementary graph of $R_{1}$, then $R_{1}$ and $R_{2}$ form a symmetric association scheme with two classes.

A relation of a non-symmetric association scheme with two classes is called a doubly regular tournament. Reid and Brown [33] proved that there exists a doubly regular tournament with $n$ vertices if and only if there exists a skew 
Hadamard matrix of order $n+1$. Thus a necessary condition is that $n \equiv 3$ $\bmod 4$.

Since a non-symmetric association scheme $\mathcal{X}$ with 3 classes is commutative, the symmetrization $\left(X,\left\{R_{0}, R_{1} \cup R_{2}, R_{3}\right\}\right)$ is also an association scheme, thus $R_{3}$ is a strongly regular graph and $R_{1}$ and $R_{2}$ are orientations of a strongly regular graph. In fact $R_{1} \cup R_{2}$ is a strongly regular graph with parameters

$$
\begin{array}{r}
(v, k, a, c)=\left(v, 2 p_{12}^{0}, p_{11}^{1}+p_{12}^{1}+p_{21}^{1}+p_{22}^{1}, p_{11}^{3}+p_{12}^{3}+p_{21}^{3}+p_{22}^{3}\right) \\
=\left(v, 2 p_{12}^{0}, 3 p_{12}^{1}+p_{22}^{1}, 2\left(p_{11}^{3}+p_{12}^{3}\right)\right) .
\end{array}
$$

In [24], we prove the following.

Lemma 1. If $A$ is the adjacency matrix of a regular directed graph (i.e., (1) is satisfied), then (2) and (3) are equivalent.

(This is also an alternative proof of the commutativity of the Bose-Mesner algebra $\mathcal{A}$ in this particular case.) A directed graph whose adjacency matrix satisfies these equations is called a normally regular digraph. The eigenvalues of a normally regular digraph have the following property.

Theorem 1 ([24]). If the adjacency matrix $A$ of a regular directed graph satisfies (2) then an eigenvalue $\theta \neq k$ lies on the circle in the complex plane with centre $\lambda-\mu$ and radius $\sqrt{k-\mu+(\lambda-\mu)^{2}}$ and $\theta+\bar{\theta}$ is an eigenvalue of $A+A^{t}$.

If $A$ satisfies all the equations (1), (2), (3) and (4) then it has four eigenvalues $\kappa$, and say $\rho, \sigma$ and $\bar{\sigma}$ with multiplicities $1, m_{1}, m_{2}$ and $m_{2}$, respectively, and the eigenvalues of $A+A^{\mathrm{t}}$ are $2 \kappa, 2 \rho$, and $\sigma+\bar{\sigma}$ with multiplicities $1, m_{1}$, and $2 m_{2}$.

For parameters $v$ and $p_{i j}^{h}, i, j, h \in\{0,1,2,3\}$ the parameters of $R_{1} \cup R_{2}$ can be computed from (6). Using standard formulas, the spectrum of $R_{1} \cup R_{2}$ can then be computed. From this it is possible to compute eigenvalues and multiplicities of $R_{1}$ (e.g. using Theorem 1). For arbitrary intersection numbers the result may be expressions for the multiplicities which are not integers.

Definition 1. We say that $v$ and $p_{i j}^{h}, i, j, h \in\{0,1,2,3\}$ form a feasible parameter set for a non-symmetric association scheme with three classes if they are non-negative integers and the multiplicities of the (four) eigenvalues computed from these intersection numbers are positive integers.

However, Bannai and Song proved that the spectrum of $A$ can be computed from the spectrum of $A+A^{\mathrm{t}}$. (We note that if the eigenvalues of $A+A^{\mathrm{t}}$ are $2 \kappa, r, s$ then either $r$ or $s$ can be split in two complex eigenvalues, if their multiplicities are even.)

Lemma 2 (Bannai and Song [2]). Suppose $A$ is an adjacency matrix of a non-symmetric relation $R_{1}$ of a 3-class association scheme. If $s$ is the eigenvalue (of multiplicity $2 m$ ) of $A+A^{t}$ that is split in two complex eigenvalues $\sigma$ and $\bar{\sigma}$ (i.e., $s=\sigma+\bar{\sigma})$ then $\sigma=\frac{1}{2}(s+i \sqrt{v \kappa / m})$. 
From the spectrum of $A$ it is possible to compute the intersection numbers.

The Hadamard product of matrices $B=\left(b_{i j}\right)$ and $C=\left(c_{i j}\right)$ is the matrix $B \circ C=\left(b_{i j} c_{i j}\right)$. Since $\left\{I, A, A^{\mathrm{t}}, J-A-A^{\mathrm{t}}-I\right\}$ is a basis of $\mathcal{A}$, it follows by considering the Hadamard product of these matrices that $\mathcal{A}$ is closed under the Hadamard product. In particular there exist numbers $q_{i j}^{h}$, for $i, j, h \in$ $\{0,1,2,3\}$, so that $E_{i} \circ E_{j}=\frac{1}{v} \sum_{h} q_{i j}^{h} E_{h}$. These numbers are called Krein parameters. It is known that each Krein parameter is a non-negative real number, see Bannai and Ito [1]. Since the Krein parameters can be computed from the spectrum of $A$, this can be used to prove non-existence for some feasible parameter sets.

Neumaier [31] found another way to exclude feasible parameter sets. Let $m_{i}$ be the rank of $E_{i}$, for $i \in\{0,1,2,3\}$. (Thus $m_{0}, \ldots, m_{3}$ are the multiplicities of eigenvalues.)

Theorem 2 ([31]). The following inequalities are satisfied for a commutative association scheme.

$$
\begin{gathered}
\sum_{h: q_{i i}^{h}>0} m_{h} \leq \frac{1}{2} m_{i}\left(m_{i}+1\right), \quad \text { for } i=0, \ldots, d, \\
\sum_{h: q_{i j}^{h}>0} m_{h} \leq m_{i} m_{j}, \quad \text { for } i, j=0, \ldots, d, i \neq j .
\end{gathered}
$$

\section{Primitive association schemes with three classes.}

We now use a computer to generate a list of all feasible parameter sets for primitive association schemes with three classes and $|X| \leq 100$. For each feasible parameter set $(v, k, a, c)$ of a strongly regular graph we investigate the feasible parameters of non-symmetric association schemes with three classes such that $R_{1} \cup R_{2}$ has parameters $(v, k, a, c)$. It follows from (6) that we need only consider parameters where $k$ and $c$ are even. It is also useful to know that the eigenvalues of $R_{1} \cup R_{2}$ are integers. This follows from the next lemma.

Lemma 3 (Goldbach and Claasen [13]). There is no non-symmetric association scheme with three classes so that $R_{1} \cup R_{2}$ has parameters $(4 c+$ $1,2 c, c-1, c)$.

In Goldbach and Claasens's terminology they proved non-existence if the strongly regular graph is pseudo-cyclic, i.e., the two non-trivial eigenvalues have the same multiplicities. It is well-known that this is equivalent to having parameters $(4 c+1,2 c, c-1, c)$, see [3].

The resulting list of feasible parameter sets is presented in Table 1 below.

The association scheme with parameter set no. 3 was constructed by Ivanov, Klin and Faradžev [22], see also [9]. Later Goldbach and Claasen [11] 
Table 1. A list of all feasible parameter sets for primitive non-symmetric 3-class association schemes with at most 100 vertices. The second column is the parameters of the strongly regular graph $R_{1} \cup R_{2}$. The third column gives information on the number of strongly regular graphs with these parameters. (In fact in some cases where we write $\geq 1$, there are several known strongly regular graph, e.g. with parameters $(100,44,18,20)$, see [25].) These numbers are from [3], [30] and [5]. In column six "NO" means that we prove non-existence of the association scheme in this paper and "no" means that non-existence follows from general results or it was proved in other papers.

\begin{tabular}{|c|c|c|c|c|c|c|}
\hline No & $\begin{array}{l}\text { Parameters } \\
\text { for } R_{1} \cup R_{2}\end{array}$ & $\begin{array}{l}\text { no. of } \\
\text { SRGs }\end{array}$ & $p_{12}^{1}$ & $p_{12}^{3}$ & exists & reference \\
\hline 1 & $(16,10,6,6)$ & 1 & 1 & 2 & no & Goldbach and Claasen [12] \\
\hline 2 & $(21,10,3,6)$ & 1 & 1 & 1 & no & Enomoto and Mena [7] \\
\hline 3 & $(36,14,4,6)$ & 180 & 0 & 2 & yes & Goldbach and Claasen [11] \\
\hline 4 & $(36,20,10,12)$ & 32548 & 3 & 2 & $\mathrm{NO}$ & Theorem 5 \\
\hline 5 & $(45,32,22,24)$ & 78 & 6 & 4 & $\mathrm{NO}$ & Theorem 4 \\
\hline 6 & $(50,42,35,36)$ & 1 & 8 & 12 & $\mathrm{NO}$ & Theorem 3 \\
\hline 7 & $(57,42,31,30)$ & 0 & 7 & 9 & no & Wilbrink and Brouwer [36] \\
\hline 8 & $(64,28,12,12)$ & $\geq 1$ & 4 & 2 & yes & Enomoto and Mena [7] \\
\hline 9 & $(64,36,20,20)$ & $\geq 1$ & 4 & 6 & $?$ & \\
\hline 10 & $(64,42,26,30)$ & $\geq 1$ & 7 & 6 & $?$ & \\
\hline 11 & $(64,42,30,22)$ & 0 & 7 & 6 & no & absolute bound \\
\hline 12 & $(81,50,31,30)$ & $\geq 1$ & 9 & 5 & $?$ & \\
\hline 13 & $(85,64,48,48)$ & $\geq 1$ & 13 & 8 & $?$ & \\
\hline 14 & $(85,70,57,60)$ & $?$ & 13 & 20 & $?$ & \\
\hline 15 & $(96,38,10,18)$ & $?$ & 3 & 4 & ? & \\
\hline 16 & $(96,50,22,30)$ & $?$ & 3 & 10 & no & Neumaier \\
\hline 17 & $(96,60,38,36)$ & $?$ & 11 & 6 & no & Krein \\
\hline 18 & $(96,76,60,60)$ & $\geq 1$ & 16 & 10 & $?$ & \\
\hline 19 & $(100,44,18,20)$ & $\geq 1$ & 3 & 6 & $?$ & \\
\hline 20 & $(100,54,28,30)$ & $\geq 1$ & 8 & 6 & $?$ & \\
\hline 21 & $(100,66,39,52)$ & 0 & 10 & 12 & no & absolute bound \\
\hline 22 & $(100,66,41,48)$ & $\geq 1$ & 8 & 16 & no & Neumaier \\
\hline 23 & $(100,66,44,42)$ & $?$ & 10 & 12 & $?$ & \\
\hline 24 & $(100,72,50,56)$ & $\geq 1$ & 13 & 12 & $?$ & \\
\hline
\end{tabular}

proved that it is the unique association scheme with these parameters. The association scheme with parameter set no. 8 was constructed by Enomoto and Mena [7]. Liebler and Mena [28] showed this scheme belongs to an infinite family of association schemes. These schemes have order $4 s^{4}$ where $s$ is a power of 2 .

These are the only known primitive non-symmetric association schemes with three classes. 
In parameter sets no. 7, 11 and 21 it is known that the strongly regular graph does not exist, see Brouwer [3]. Thus the 3-class association scheme does not exist in these cases.

In parameter set no. 17 some of the Krein parameters are negative. Thus this case is excluded. The multiplicities of eigenvalues for parameter sets no. 16 and 22 do not satisfy Neumaier's condition.

We will now use computers to prove non-existence of association schemes with parameter sets no. 4, 5 and 6 . We use two different techniques.

For parameter sets no. 5 and 6 , the computation is based on the classification of all strongly regular graphs which have the same parameters as the strongly regular graph $R_{3}$ (assuming that $\left(X,\left\{R_{0}, R_{1}, R_{2}, R_{3}\right\}\right)$ is the required association scheme). For a given graph $R_{3}$ we try to construct $R_{1}$ by orienting the complement of $R_{3}$. We first consider orientation of edges of the complement of $R_{3}$ incident with a fixed vertex $x$. We let $N^{+}(x)$ denote the outneighbours of $x$ in $R_{1}$ and we let $N_{2}(x)$ denote the vertices at distance 2 from $x$ in $R_{3}$. Then a candidate for $N^{+}(x)$ consists of exactly half of the vertices in $N_{2}(x)$. But it also has some other properties. Let $x_{1}, \ldots, x_{k}$ be the neighbours of $x$ in $R_{3}$ and $S_{i}$ denote the set of neighbours of $x_{i}$ in $N_{2}(x)$, for $i=1, \ldots, k$. Then a candidate for $N^{+}(x)$ must satisfy $\left|S_{i} \cap N^{+}(x)\right|=p_{13}^{3}=\frac{1}{2}\left|S_{i}\right|$. It also satisfies that the subgraph of $R_{3}$ induced by $N^{+}(x)$ is regular of degree $p_{13}^{1}$ and the subgraph induced by $N_{2}(x) \backslash N^{+}(x)$ is regular of degree $p_{23}^{2}=p_{13}^{1}$. When we have computed the list of all candidates for $N^{+}(x)$ for every vertex $x$, we try if it is possible to combine these orientations in such a way that for any two vertices $x$ and $y$ the orientation of the edges incident with $x$ and the orientation of the edges incident with $y$ should agree on the orientation of the edge $\{x, y\}$ if $x$ and $y$ are non-adjacent in $R_{3}$, and they should satisfy that for all $i, j$ the number of vertices $z$ so that $(x, z) \in R_{i}$ and $(z, y) \in R_{j}$ is exactly $p_{i j}^{h}$ where $(x, y) \in R_{h}$.

For parameters no. $6, R_{3}$ is a strongly regular graph with parameters $(50,7,0,1)$, i.e., it is the Hoffman-Singleton graph, see [16]. This case can be excluded by investigating possible orientations of the complement of the Hoffman-Singleton graph.

Theorem 3. There is no non-symmetric association scheme with three classes where $R_{3}$ is the Hoffman-Singleton graph.

Proof. Suppose that there exists a non-symmetric association scheme with three classes where $R_{3}$ is the Hoffman-Singleton graph. When applying the method described above we may use that the Hoffman-Singleton graph has a large group of automorphisms. Computations using this group are done in GAP [10] with GRAPE [34] and nauty [29]. Other computation are done in a C-program.

Let $x$ be a vertex and let $x_{1}, \ldots, x_{7}$ be the neighbours of $x$ in $R_{3}$. Let $S_{i}$ be the set of neighbours of $x_{i}$ other than $x$, for $i=1, \ldots, 7$. Let $N^{+}(x)$ be the set out-neighbours of $x$ in $R_{1}$. Then $N^{+}(x)$ is a set of 21 vertices in the set $N_{2}(x)=S_{1} \cup \ldots \cup S_{7}$ of vertices at distance 2 from $x$, and $\left|S_{i} \cap N^{+}(x)\right|=$ 
$p_{13}^{3}=3$, for $i=1, \ldots, 7$. The subgraph of $R_{3}$ spanned by $N^{+}(x)$ is regular of degree $p_{13}^{1}=4$. The complement of $N^{+}(x)$ in $S_{1} \cup \ldots \cup S_{7}$ is the set of in-neighbours of $x$ in $R_{1}$ and this set also spans a 4-regular subgraph of $R_{3}$.

A computer enumeration shows that there are exactly 1140 subsets of $N_{2}(x)$ with the properties required for $N^{+}(x)$. These 1140 subsets form three orbits under the action of the subgroup of the automorphism group of the Hoffman-Singleton graph stabilizing the vertex $x$.

Thus we need only consider three possibilities for $N^{+}(x)$, but then we must consider all 1140 candidates $N^{+}(y)$ for any other vertex $y$. It turns out that we only need to consider orientations of edges incident with $x, x_{1}, \ldots, x_{5}$. These edges must by oriented such that $\left|N^{+}(x) \cap N^{+}\left(x_{i}\right)\right|=p_{12}^{3}=12$, as $\left(x, x_{i}\right) \in R_{3},\left|N^{+}\left(x_{i}\right) \cap N^{+}\left(x_{j}\right)\right|=p_{12}^{1}=p_{12}^{2}=8$, as $\left(x_{i}, x_{j}\right) \notin R_{3}$, and such that $x_{j} \in N^{+}\left(x_{i}\right)$ if and only if $x_{i} \notin N^{+}\left(x_{j}\right)$.

A computer search shows that there are no orientations of all edges incident with $x, x_{1}, x_{2}, x_{3}, x_{4}$ and $x_{5}$ that satisfy these conditions. Thus the required association scheme does not exist.

For parameters no. $5, R_{3}$ is a strongly regular graph with parameters $(v, k, a, c)=(45,12,3,3)$.

Coolsaet, Degraer and Spence [5], have shown that there are exactly 78 strongly regular graphs with these parameters. Thus the method from the previous theorem can be applied to each of these 78 graphs.

Theorem 4. There is no primitive non-symmetric association scheme with three classes with parameter set no. 5 .

Proof. Suppose that there exists such an association scheme. Let $x$ be a vertex and let $x_{1}, \ldots, x_{12}$ be the neighbours of $x$ in $R_{3}$. Let $S_{i}$ be the set of neighbours of $x_{i}$ at distance 2 from $x,\left|S_{i}\right|=k-a-1=8$, for $i=1, \ldots, 12$. Let $N^{+}(x)$ be the set out-neighbours of $x$ in $R_{1}$. Then $N^{+}(x)$ is a set of 16 vertices in the set $N_{2}(x):=S_{1} \cup \ldots \cup S_{12}$, and $\left|S_{i} \cap N^{+}(x)\right|=p_{13}^{3}=4$, for $i=1, \ldots, 12$. The subgraph of $R_{3}$ spanned by $N^{+}(x)$ is regular of degree $p_{13}^{1}=3$.

The computer search shows that if $N$ is a set with $\left|S_{i} \cap N\right|=4$, for $i=1, \ldots, 12$, and in which every vertex has degree at most 3 then $N$ is 3-regular and the subgraph of $R_{3}$ spanned by $N_{2}(x) \backslash N$ is also 3-regular.

The number of such sets $N$ depend on the graph and the vertex $x$. The largest number of sets is 396 , which appear in the graph with a rank 3 automorphism group.

44 of the 78 candidates for $R_{3}$ can be excluded because, for at least one vertex $x$, there is no such set $N$.

For each of the other 34 graphs we find by computer search a set $W$ of at most 8 vertices so that there is no combination of orientations of edges in the complement of $R_{3}$ incident with $w$, for each $w \in W$ that satisfies the required properties. (This search took 45 minutes on a $2.4 \mathrm{GHz}$ PC.)

Thus an association scheme with parameter set no. 5 does not exist. 
For parameter set no. 4 (and for one case of imprimitive association schemes, see section 4) we use a different computer search technique. This does not depend on characterization of strongly regular graphs.

We use an orderly generation algorithm (see Faradžev [8] or Read [32]) to search for the matrix $B=3 A_{3}+2 A_{2}+A_{1}$, where $A_{1}, A_{2}, A_{3}$ are adjacency matrices of the relations $R_{1}, R_{2}, R_{3}$ of the required association scheme. Recall that for $i \in\{1,2,3\}$ we define $i^{\prime} \in\{1,2,3\}$ so that $R_{i}^{\mathrm{t}}=R_{i^{\prime}}$. In our usual enumeration of relations this means that $1^{\prime}=2,2^{\prime}=1$ and $3^{\prime}=3$, but in the first application of the algorithm (Theorem 5) we use a different enumeration (where $1^{\prime}=1,2^{\prime}=3$ and $3^{\prime}=2$ ).

We want the vertices to be enumerated so that the matrix $B$ is in maximal form, i.e., the sequence obtained by reading the entries of the first row followed by the entries of the second row, etc., is as large as possible (in the lexicographic order) among all enumerations of the vertices.

Suppose that the first $r-1$ rows of the matrix $B=\left(b_{i j}\right)$ has been filled in. We then investigate all possible ways to fill in row $r$ with 0 on the diagonal entry, $p_{11^{\prime}}^{0}$ entries with 1's, $p_{22^{\prime}}^{0}$ entries with 2's, and $p_{33^{\prime}}^{0}$ entries with 3's in such a way that

- the first $r-1$ entries are in accordance with the entries of column $r$ of the previous rows.

- for each $x<r$ the number of columns $s$, so that $b_{x s}=i$ and $b_{r s}=j^{\prime}$ is exactly $p_{i j}^{h}$, where $b_{x r}=h$.

- the matrix is still in maximal form.

For each possible way to fill row $r$ we repeat the procedure for row $r+1$.

Theorem 5. There is no primitive non-symmetric association scheme with three classes with parameter set no. 4 .

Proof. As described above, we search for the matrix $B=3 A_{3}+2 A_{2}+A_{1}$.

In turns out that with the maximality condition on the matrix and for this particular parameter set it is convenient to enumerate the relations so that $R_{1}$ is symmetric and $R_{2}^{\mathrm{t}}=R_{3}$. Thus the first row of $B$ should consist one 0 followed by $p_{33^{\prime}}^{0}=10$ entries with 3's followed by $p_{22^{\prime}}^{0}=10$ entries with 2's and finally $p_{11^{\prime}}^{0}=15$ entries with 1 's.

When using the algorithm described above we find that the number of ways to fill in the first $r$ rows is 1, 1, 100, 24161, 205671, 1116571, 52650, $39,0, \ldots, 0$, for $r=1, \ldots, 36$. Thus the required association scheme does not exist. (This search took 81 minutes on a $2.4 \mathrm{GHz}$ PC.) 


\section{Imprimitive association schemes with three classes.}

\subsection{General results}

If $R_{3}$ is connected but $R_{1}$ and $R_{2}$ are disconnected then each connected component of $R_{1}$ is a doubly regular tournament on $2 p_{12}^{0}+1$ vertices. Thus the study of these schemes reduces to the study of doubly regular tournaments.

We will thus assume that $R_{1}$ and $R_{2}$ are connected and $R_{3}$ is disconnected. Then $R_{3}$ consists of $m$ copies of a complete graph on $r$ vertices, for some constants $m$ and $r$. We denote this graph by $m \circ K_{r}$. Then $R_{1}$ is an orientation of the complement $\overline{m \circ K_{r}}$. The vertex set of $\overline{m \circ K_{r}}$ is partitioned in $m$ independent sets of size $r$, denoted by $V_{1}, \ldots, V_{m}$.

In [26] we introduce the following family of graphs that do not necessarily satisfy all the conditions on a relation of a non-symmetric association scheme with three classes. We say that a directed graph is a doubly regular $(m, r)$ team tournament if it is an orientation of $\overline{m \circ K_{r}}$ with adjacency matrix $A$ satisfying (1) and (4) in Section 2.

In $[26]$ we give a combinatorial proof of the following, i.e., we do not use eigenvalues.

Theorem 6 (Jørgensen, Jones, Klin and Song [26]). Every doubly regular $(m, r)$-team tournament is of one of the following types.

1. For every pair $i, j$ either all the edges between $V_{i}$ and $V_{j}$ are directed from $V_{i}$ to $V_{j}$, or they are all directed from $V_{j}$ to $V_{i}$. The graph with vertices $v_{1}, \ldots, v_{m}$ and an edge directed from $v_{i}$ to $v_{j}$ if edges are directed from $V_{i}$ to $V_{j}$ is a doubly regular tournament.

2. For every vertex $x \in V_{i}$, exactly half of the vertices in $V_{j}(j \neq i)$ are out-neighbours of $x$, and $\alpha=\beta=\frac{(m-2) r}{4}$, and $\gamma=\frac{(m-1) r^{2}}{4(r-1)}$.

3. For every pair $\{i, j\}$ either $V_{i}$ is partitioned in two sets $V_{i}^{\prime}$ and $V_{i}^{\prime \prime}$ of size $\frac{r}{2}$ so that all edges between $V_{i}$ and $V_{j}$ are directed from $V_{i}^{\prime}$ to $V_{j}$ and from $V_{j}$ to $V_{i}^{\prime \prime}$, or similarly with $i$ and $j$ interchanged. The parameters are $\alpha=\frac{(m-1) r}{4}-\frac{3 r}{8}, \beta=\frac{(m-1) r}{4}+\frac{r}{8}, \gamma=\frac{(m-1) r^{2}}{8(r-1)}$.

A graph of type 3 can not be a relation of an association scheme. In this case 8 divides $r$ and $4(r-1)$ divides $m-1$. We do not know if any graph of this type exists.

Every graph of type 1 or type 2 is a relation of a non-symmetric association scheme with 3 classes. The results for these types where first proved by Goldbach and Claasen [14].

Clearly, the graph of type 1 exists if and only if a doubly regular tournament of order $m$ exists. Thus in the remaining part this section we will only consider graphs of type 2 . 


\subsection{Association schemes of type 2}

We first show that a graph of type 2 is a relation of a non-symmetric association scheme with 3 classes. This is done by proving that (2) and (3) are satisfied.

Lemma 4. Let $A$ be the adjacency matrix of a doubly regular $(m, r)$-team tournament of type 2. Then A satisfies (2) and (3) with

- $\lambda=\alpha=\frac{(m-2) r}{4}$ and

- $\mu=\frac{(m-1) r(r-2)}{4(r-1)}$.

In particular if $m=r$ then $\lambda=\mu=\frac{m(m-2)}{4}$.

Proof. Let $x \in V_{i}$ and $y \in V_{j}, i \neq j$, and suppose that there is an edge directed from $x$ to $y$. Then $x$ has $\kappa-\frac{r}{2}$ out-neighbours outside $V_{i} \cup V_{j}, \alpha$ of these are in-neighbours of $y$ and the remaining $\kappa-\frac{r}{2}-\alpha$ are out-neighbours of $y$. Thus $\lambda=\kappa-\frac{r}{2}-\alpha=\frac{(m-2) r}{4}$, since $\kappa=\frac{(m-1) r}{2}$.

Similarly, for $x, y \in V_{i}$, we get $\mu=\kappa-\gamma=\frac{(m-1) r(r-2)}{4(r-1)}$. Thus (2) is satisfied. Equation (3) can be proved in a similar way, or by applying Lemma 1.

Since the parameters of a graph of type 2 are integers, it follows that $r$ is even and $r-1$ divides $m-1$. Using eigenvalues, it can be shown that $m$ is even, see [26] or Goldbach and Claasen [14].

Existence in the case $r=2$ is equivalent to existence of a doubly regular tournament of order $m-1$.

Theorem 7 ([26]). If there exists a doubly regular $(m, 2)$-team tournament $\Gamma$ then 4 divides $m$ and the out-neighbours of a vertex in $\Gamma$ span a doubly regular tournament of order $m-1$.

Conversely, for every doubly regular tournament $T$ of order $m-1$, there exists a doubly regular $(m, 2)$-team tournament $\Gamma$, such that for some vertex $x$ in $\Gamma$ the out-neighbours of $x$ span a subgraph isomorphic to $T$.

No schemes with $4 \leq r<m$, where $r-1$ divides $m-1$ are known.

We will now consider the case $m=r$. By Lemma 4, the directed graph is then a normally regular digraph with $\mu=\lambda$. Such digraphs are also known as doubly regular asymmetric digraphs. These graphs were introduced and studied in a series of papers by N. Ito [18], [19], [20] and [21] and also studied by Ionin and Kharaghani [17].

The first non-trivial case of an association scheme of type 2 with $m=r$ is for $m=4$. In this case there exist two non-isomorphic schemes. One of these schemes has an automorphism group of rank 4, i.e., the group acts transitively 
on the vertices and the stabilizer of a vertex $x$ has four orbits: $\{x\}$, the set of out-neighbours of $x$, the set of in-neighbours of $x$ and the set of vertices not adjacent to $x$. Any doubly regular asymmetric digraph with automorphism group of rank 4 is a relation of a non-symmetric association scheme with 3 classes. Ito [19] has proved that a non-symmetric 3-class association scheme with $\mu=\lambda$ does not satisfy the feasibility condition. Thus a doubly regular asymmetric digraph with automorphism group of rank 4 is a relation of an imprimitive non-symmetric 3-class association scheme of type 2 with $m=r$ (as $\mu=\lambda$ ). In this case Ito [19] has proved that $m=r$ is a power of 2 . He also claims to have proved that the only possibility is $m=4$. But the proof of this does not seem to be correct and in fact Ito in his paper gives an example of a vertex transitive scheme with $m=r=8$. According to computations in GAP [10] using share package GRAPE [34] with nauty [29] the automorphism group of this scheme has rank 4.

We will now consider the links between such association schemes and a special case of some well-known structures.

Definition 2. An Hadamard matrix $H$ of order $n$ is an $n \times n$ matrix in which every entry is either 1 or -1 and $H H^{t}=n I$.

An Hadamard matrix $H$ of order $m^{2}$ is said to be Bush-type if $H$ is block matrix with $m \times m$ blocks $H_{i j}$ of size $m \times m$ such that $H_{i i}=J_{m}$ and $H_{i j} J_{m}=$ $J_{m} H_{i j}=0$, for $i \neq j$.

Theorem 8. An imprimitive 3-class association scheme of type 2 and with $r=m$ is equivalent to a Bush-type Hadamard matrix of order $m^{2}$ with the property that $H_{i j}=-H_{j i}^{t}$, for all pairs $i, j$ with $i \neq j$.

Proof. Let $A$ be an adjacency matrix of relation $R_{1}$, for some imprimitive 3 -class association scheme of type 2 and with $r=m$. We may assume that vertices are enumerated such that the vertices in $V_{i}$ corresponds to columns/rows $m i-m+1, \ldots, m i$. Let $H=J_{m^{2}}-2 A$. Then $H$ is partitioned in blocks $H_{i j}$ of size $m \times m$ corresponding to the partition of vertices in sets $V_{1}, \ldots, V_{m}$. Clearly $H_{i i}=J_{m}$ and since a vertex in $V_{i}$ has exactly $\frac{m}{2}$ out-neighbours and $\frac{m}{2}$ in-neighbours in $V_{j}, H_{i j} J_{m}=J_{m} H_{i j}=0$.

From (1) and (2) we get (since $\kappa=\frac{m(m-1)}{2}$ and $\mu=\lambda=\frac{m(m-2)}{4}$ )

$H H^{\mathrm{t}}=\left(J_{m^{2}}-2 A\right)\left(J_{m^{2}}-2 A^{\mathrm{t}}\right)=\left(m^{2}-4 \kappa\right) J_{m^{2}}+4\left(\kappa I+\mu\left(J_{m^{2}}-I\right)\right)=m^{2} I$.

Thus $H$ is an Hadamard matrix.

Conversely, suppose that $H$ is a Bush-type Hadamard matrix which is skew in the sense that $H_{i j}=-H_{j i}^{\mathrm{t}}$, for $i \neq j$.

Let $A=\frac{1}{2}(J-H)$, where $J=J_{m^{2}}$. Then $A$ is a $\{0,1\}$ matrix. Since $H$ is Bush-type it has exactly $m+(m-1) \frac{m}{2}$ entries equal to 1 and $(m-1) \frac{m}{2}$ entries equal to -1 in each row. Thus $H J=m J$ and the transposed equation is $J H^{\mathrm{t}}=m J$. Similarly $J H=m J$. Thus $A J=J A=\frac{m(m-1)}{2} J$ and 


$$
A A^{\mathrm{t}}=\frac{1}{4}(J-H)\left(J-H^{\mathrm{t}}\right)=\frac{m(m-2)}{4} J+\frac{m^{2}}{4} I .
$$

We see that (1) and (2) are satisfied. Equation (3) can be proved in a similar way, or by applying Lemma 1.

Let $K$ denote the block diagonal matrix with diagonal blocks equal to $J_{m}$. Then the Bush-type property of $H$ implies that $H K=m K$ and the skew property of $H$ implies that $H+H^{\mathrm{t}}=2 K$. Thus $H^{2}=H\left(2 K-H^{\mathrm{t}}\right)=$ $2 m K-m^{2} I$, and so

$$
A^{2}=\frac{1}{4}(J-H)^{2}=\frac{1}{4}\left(m(m-2) J+2 m K-m^{2} I\right) .
$$

Since $J-I-A-A^{\mathrm{t}}=K-I$, it follows that (4) is satisfied with $\alpha=\beta=\frac{m(m-2)}{4}$ and $\gamma=\frac{m^{2}}{4}$.

Kharaghani [27] proved that if there exists an Hadamard matrix of order $m$ then there exists a Bush-type Hadamard matrix of order $m^{2}$.

Ionin and Kharaghani [17] modified this construction and proved that if there exists an Hadamard matrix of order $m$ then there exists a Bushtype Hadamard matrix of order $m^{2}$, which has the skew property required in Theorem 8.

Thus in many cases with $m=r$ a multiple of 4 , an association scheme can be constructed.

The case with $m=r$ congruent to 2 modulo 4 seems to be more difficult and no general constructions are known. But in the special case $m=r=6$ we may apply the orderly generation algorithm described before Theorem 5 .

The number of ways to fill the first $s$ rows is $1,1,4,12,8,6,29077$, 76216458 , for $s=1,2, \ldots, 8$. (Note that the first six rows correspond to a connected component of the undirected graph $R_{3}$.) We estimate that a complete search through all 76 million ways to fill the first 8 rows would take several years. But we guessed (especially because there are no such schemes with a rank 4 group) that if a scheme exists then there are many schemes and so a partial search may lead to a least one scheme.

Probably starting a complete search and let the computer run until a scheme is discovered is not an optimal strategy. Instead, we chose 2405 cases randomly among all ways to fill 8 rows. This search gave 47 ways to fill 13 rows but no ways to fill 14 rows. The idea is now to do a complete search in the "neighbourhood" of the most successful 8-row matrices, where the neighbourhood of an 8-row matrix is the set of all 8-row matrices with which it has the first 7 rows in common. This search lead to two association schemes. A repetition (with another set of randomly chosen 8-row matrices) gave two other schemes.

Thus we have:

Theorem 9. There exist at least four imprimitive non-symmetric 3-class association schemes of type 2 with $m=r=6$. 
Each of these four schemes have a trivial automorphism group.

The computation of automorphism groups can be done in GAP [10] using share package GRAPE [34] with nauty [29].

The adjacency matrix of $R_{1}$ is listed in Table 2 for one of these four schemes. Note that we have reordered rows and columns so that the imprimitive structure is clear. The matrix is not in maximal form in this ordering (even with the 3's and 2's that have been replaced by 0's).

A Bush-type Hadamard matrix of order 36 was first constructed by Janko [23]. But a "skew" Bush-type Hadamard matrix was not previously known. Bussemaker, Haemers and Spence [4] proved that a symmetric Bushtype Hadamard matrix of order 36 does not exist.

\section{Concluding remarks}

We have seen in Section 3 that very few primitive non-symmetric 3-class association schemes are known. In fact (except for the first 8 cases) the problem of existence is still open for the majority of feasible parameter sets. We do not expect that the orderly generation algorithm described in Section 3 can be applied to the remaining open cases in the primitive case. However, the other technique using information about the strongly regular graph obtained by merging the non-symmetric relations may still be used in some particular cases. It would also be very useful to develop new computer aided search methods or even some computer free methods. It could also be interesting to get information about existence of association schemes with a given group of automorphisms.

In the imprimitive case the situation is quite different. Here we have many constructions, especially because of the connection to Hadamard matrices. The most interesting open problem in the imprimitive case is whether there exist association schemes of type 2 with $4 \leq r<m$. The smallest feasible case is $r=4, m=10$ with order 40 . We tried to attack this problem with the orderly generation algorithm, but it seems that the search space is too large. However, it may be that the algorithm can be improved so that this problem can be solved. But it seems that it is easier to solve the still open problem of complete enumeration of association schemes in the case $m=r=6$.

\section{Acknowledgment}

The author wishes to thank Prof. Mikhail Klin for some very helpful discussions and suggestions on this paper and the research reported in it.

The author is pleased to acknowledge Prof. Bruno Buchberger and the coordinators of the Special Semester on Gröbner Bases (February 1 - July 31, 2006), organized by RICAM , Austrian Academy of Sciences, and RISC, Johannes Kepler University, Linz Austria for their attention to this project. 
Table 2. Matrix of a 3-scheme with $m=r=6$

\begin{tabular}{|c|c|c|c|c|c|}
\hline $\begin{array}{llllll}0 & 0 & 0 & 0 & 0\end{array}$ & $\begin{array}{lllll}1 & 1 & 0 & 0 & 0\end{array}$ & & & & \\
\hline 000000 & 10100 & $\begin{array}{lllll}0 & 0 & 1 & 1 & 0\end{array}$ & 100110 & 110100 & \\
\hline $\begin{array}{lllll}0 & 0 & 0 & 0 & 0\end{array}$ & 100011 & 100101 & $\begin{array}{llllll}0 & 1 & 0 & 1 & 0 & 1\end{array}$ & $\begin{array}{lllllll}0 & 0 & 1 & 1 & 1 & 0\end{array}$ & 11010 \\
\hline $\begin{array}{lllll}0 & 0 & 0 & 0 & 0\end{array}$ & 0111010 & $\begin{array}{llll}10 & 0 & 1\end{array}$ & $\begin{array}{llllll}1 & 0 & 0 & 0 & 1 & 1\end{array}$ & $\begin{array}{lllllll}0 & 0 & 0 & 1 & 1 & 1\end{array}$ & \\
\hline 0000 & $\begin{array}{lllll}0 & 0 & 1 & 1 & 1\end{array}$ & 11010 & & 1 & \\
\hline 0000 & 01101 & $\begin{array}{lllll}0 & 1 & 1 & 0 & 1\end{array}$ & 0111100 & $\begin{array}{llll}0 & 0 & 1 & 1\end{array}$ & \\
\hline & & & & & \\
\hline & & & & & \\
\hline & & & & & \\
\hline & & & & & \\
\hline & & & & & \\
\hline & & & & & \\
\hline & & & & & \\
\hline & & & & & \\
\hline & & & & & \\
\hline & & & & & \\
\hline & & & & & \\
\hline & & $\begin{array}{llll}0 & 0 & 0 & 0\end{array}$ & & & \\
\hline & & & & & \\
\hline & & & & & \\
\hline & & & & & \\
\hline & & & & & \\
\hline & & & & & \\
\hline & & 101 & & & \\
\hline & & & & & \\
\hline & & & & & \\
\hline & & & & & \\
\hline & & & & & \\
\hline & & & & & \\
\hline & & & & & \\
\hline & & & & & \\
\hline & & & & & \\
\hline & & & & & \\
\hline & & 111 & & & \\
\hline & & & & & \\
\hline
\end{tabular}




\section{References}

1. E. Bannai and T. Ito, Algebraic Combinatorics. I. Benjamin/Cumming, Menlo Park, 1984.

2. E. Bannai and S.-Y. Song, Character tables of fission schemes and fusion schemes, European J. Combin. 14 (1993), 385-396.

3. A. E. Brouwer, Strongly regular graphs, in: The CRC Handbook of Combinatorial Designs (eds.: Colbourn and Dinitz), 667-685, CRC Press, 1996.

4. F. C. Bussemaker, W. Haemers, and E. Spence, The search for pseudo orthogonal latin squares of order six, Designs, Codes and Cryptography 21 (2000), $77-82$.

5. K. Coolsaet, J. Degraer and E. Spence, The strongly regular $(45,12,3,3)$ graphs, Electron. J. Combin. 13 (2006), no. 1, Research Paper 32, 9 pp.

6. E. R. van Dam, Three-class association schemes, J. Algebraic Combin. 10 (1999), 69-107.

7. H. Enomoto and R. A. Mena, Distance-regular digraphs of girth 4, J. Combin. Th., Ser. B 43 (1987), 293-302.

8. I. A. Faradžev, Constructive enumeration of combinatorial objects, Problèmes combinatoires et théorie des graphes. Colloq. Internat. CNRS, 260, CNRS, Paris (1978) 131-135.

9. I. A. Faradžev, M. H. Klin and M. E. Muzichuk, Cellular rings and groups of automorphisms of graphs, in: Investigations in algebraic theory of combinatorial objects, edited by I. A. Faradžev, A. A. Ivanov, M. H. Klin and A. J. Woldar, Kluwer 1994.

10. The GAP Group, GAP - Groups, Algorithms, and Programming, Version 4.4.9; 2006, (http://www.gap-system.org).

11. R. W. Goldbach and H. L. Claasen, A primitive non-symmetric 3-class association scheme on 36 elements with $p_{11}^{1}=0$ exists and is unique, Europ. J. Combin. 15 (1994), 519-524.

12. R. W. Goldbach and H. L. Claasen, On splitting the Clebsch graph, Indag. Math. (N.S.) 5 (1994), 285-290.

13. R. W. Goldbach and H. L. Claasen, Feasibility conditions for non-symmetric 3-class association schemes, Discrete Math. 159 (1996), 111-118.

14. R. W. Goldbach and H. L. Claasen, The structure of imprimitive non-symmetric 3-class association schemes, Europ. J. Combin. 17 (1996), 23-37.

15. D. G. Higman, Coherent configurations, Geom. Dedicata 4 (1975) 1-32.

16. A. J. Hoffman and R. R. Singleton, On Moore graphs with diameters 2 and 3. IBM J. Res. Develop. 4 (1960), 497-504.

17. Y. J. Ionin and H. Kharaghani, Doubly regular digraphs and symmetric designs, J. Combin. Th., Ser. A 101 (2003) 35-48.

18. N. Ito, Doubly regular asymmetric digraphs, Discr. Math. 72 (1988) 181-185.

19. N. Ito, Automorphism groups of DRADs, Group Theory (Singapore, 1987), de Gruyter, Berlin (1989), 151-170.

20. N. Ito, Doubly regular asymmetric digraphs with rank 5 automorphism groups, Groups-Korea 1988), Lecture Notes in Math., 1398, Springer (1989), 94-99.

21. N. Ito, On spectra of doubly regular asymmetric digraphs of RH-type, Graphs Combin. 5 (1989) 229-234.

22. A.A.Ivanov, M.H.Klin and I.A.Faradžev, The primitive representations of the non-abelian simple groups of order less than $10^{6}$. Part 2 (Russian). Preprint, Moscow, Institute for System Studies, 1984, 76 pp. 
23. Z. Janko, The existence of a Bush-type Hadamard matrix of order 36 and two new infinite classes of symmetric designs, J. Combin. Th., Ser A 95 (2001) $360-364$.

24. L. K. Jørgensen, Normally regular digraphs, preprint R-94-2023, Institute for Electronic Systems, Aalborg University, 1994.

25. L. K. Jørgensen and M. H. Klin, Switching of edges in strongly regular graphs. I. A family of partial difference sets on 100 vertices. Electron. J. Combin. 10 (2003), Research Paper 17, 31 pp.

26. L. K. Jørgensen, G. A. Jones, M. H. Klin and S. Y. Song, Normally regular digraphs, association schemes and related combinatorial structures. In preparation.

27. H. Kharaghani, On the twin designs with the Ionin-type parameters, Elec. J. Combin. 7 (2000), R1.

28. R. A. Liebler and R. A. Mena, Certain Distance regular digraphs and related rings of characteristic 4, J. Combin. Th., Ser B 47 (1988), 111-123.

29. B.D. McKay, nauty user's guide (version 1.5), Technical report TR-CS-90-02, Australian National University, Computer Science Department, ANU, 1990.

30. B. D. McKay and E. Spence, Classification of regular two-graphs on 36 and 38 vertices, Australas. J. Comb 24 (2001), 293-300.

31. A. Neumaier, New inequalities for the parameters of an association scheme, Combinatorics and graph theory (Calcutta, 1980), 365-367, Lecture Notes in Math., 885, Springer, Berlin-New York, 1981.

32. R. C. Read, Every one a winner or How to avoid isomorphism search when cataloguing combinatorial configurations, Annals of Discr. Math. 2 (1978) 107120.

33. K. B. Reid and E. Brown, Doubly regular tournaments are equivalent to skew Hadamard matrices. J. Combinatorial Theory, Ser. A 12 (1972), 332-338.

34. L. H. Soicher. GRAPE: a system for computing with graphs and groups. In: Groups and Computation (eds.: Finkelstein and Kantor), volume 11 of DIMACS Series in Discrete Mathematics and Theoretical Computer Science, pages 287291. American Mathematical Society, 1993.

35. S.-Y. Song, Class 3 association schemes whose symmetrization have two classes, J. Combin. Th. Ser. A 70 (1995), 1-29.

36. H. A. Wilbrink and A. E. Brouwer, A $(57,14,1)$ strongly regular graph does not exist, Indag. Math. 45 (1983), 117-121. 